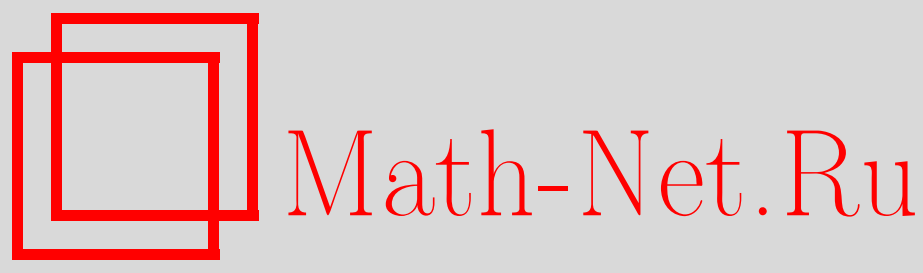

М. Э. Муминов, Формула для числа собственных значений трехчастичного оператора Шредингера на решетке, ТMФ, 2010, том 164, номер 1, 46-61

DOI: https://doi.org/10.4213/tmf6523

Использование Общероссийского математического портала Math-Net.Ru подразумевает, что вы прочитали и согласны с пользовательским соглашением http://www.mathnet.ru/rus/agreement

Параметры загрузки:

IP: 54.237 .206 .68

26 апреля 2023 г., 16:05:46

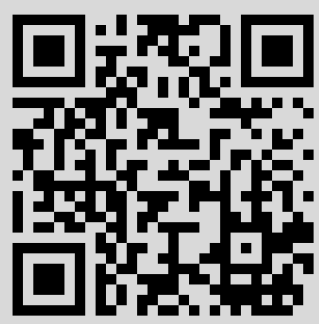




\title{
ФИЗИКА
}

Том 164, № 1

июль, 2010

2010 г.

\author{
М.Э. Муминов ${ }^{* \dagger}$
}

\section{ФОРМУЛА ДЛЯ ЧИСЛА СОБСТВЕННЫХ ЗНАЧЕНИЙ ТРЕХЧАСТИЧНОГО ОПЕРАТОРА ШРЕДИНГЕРА НА РЕШЕТКЕ}

Рассмотрена система из трех произвольных квантовых частиц на трехмерной решетке, взаимодействующих посредством короткодействующих потенциалов притяжения. Получена формула для числа собственных значений, лежащих в произвольном интервале вне существенного спектра трехчастичного дискретного оператора Шредингера. Найдено достаточное условие конечности дискретного спектра. Приведен пример применения полученных результатов.

Ключевые слова: дискретный и существенный спектры, оператор Шредингера, положительный оператор, компактный оператор.

\section{1. ВВЕДЕНИЕ}

Существование бесконечного набора собственных значений трехчастичного оператора Шредингера и асимптотика числа собственных значений были получены сначала в статье [1], затем - в работе [2], а также, в случае оператора на решетке, в работах [3]-[5]. В этих работах для $z$, лежащих левее существенного спектра оператора Шредингера, построен компактный оператор $A(z)$ такой, что $N(z)=n(1, A(z))$, где $N(z)$ - число собственных значений оператора Шредингера, лежащих левее $z$, и $n(\lambda, A)$ - число собственных значений оператора $A(z)$, лежащих правее $\lambda$. Этот оператор играет важную роль для получения указанной асимптотики. Заметим, что, в отличие от существенного спектра трехчастичного непрерывного оператора Шредингера, существенный спектр $\sigma_{\mathrm{ess}}(H(K))$ трехчастичного дискретного оператора Шредингера $H(K)$, где $K$ - полный квазиимпульс системы трех частиц, может содержать лакуны, т.е. существует интервал $(a, b)$,

$$
\inf \sigma_{\mathrm{ess}}(H(K)) \leqslant a<b \leqslant \sup \sigma_{\mathrm{ess}}(H(K)),
$$

такой, что $(a, b) \cap \sigma_{\mathrm{ess}}(H(K))=\varnothing($ см. работу [6]).

\footnotetext{
* Самаркандский государственный университет им. Алишера Навои, Самарканд, Узбекистан. E-mail: mmuminov@mail.ru

${ }^{\dagger}$ Department of Mathematics, Dogus University, Istanbul, Turkey
} 
Отметим, что формула $N(z)=n(1, A(z))$ справедлива только при $z$, лежащих левее существенного спектра; она позволяет утверждать, что множество точек дискретного спектра, лежащих левее существенного спектра, конечно, если компактен оператор $A(\tau)$, где $\tau=\inf \sigma_{\text {ess }}(H(K))$ (см. лемму 8 работы [7]). Аналогичную формулу для $z$, лежащих на лакуне существенного спектра, невозможно получить вариационным методом, примененным в работах [1], [5]. Поэтому возникает интересная задача - для $z$, лежащих на лакуне существенного спектра, вывести аналогичную формулу, позволяющую найти асимптотику или доказать конечность множества точек дискретного спектра на лакуне.

В настоящей работе рассматривается система трех произвольных квантовых частиц на трехмерной решетке, взаимодействующих с помощью короткодействующих потенциалов притяжения, и проводится анализ дискретного спектра соответствующего трехчастичного дискретного оператора Шредингера $H(K)$. Точнее, получена формула для числа собственных значений, лежащих в произвольном интервале вне существенного спектра этого оператора, и для $z$, лежащих левее существенного спектра $H(K)$, и на лакуне существенного спектра (если такая лакуна существует). Построен самосопряженный компактный оператор $\mathbf{T}(z)$ такой, что $N(z)=n(1, \mathbf{T}(z))$ при $z<\inf \sigma_{\text {ess }}(H(K))$. Также найдено достаточное условие конечности множества точек дискретного спектра.

Отметим, что в работе [8] получены некоторые оценки и равенства для числа собственных значений самосопряженных ограниченных снизу оператор-функций, удовлетворяющих определенным условиям. В работе [9] выведена формула для числа собственных значений, лежащих в произвольном интервале вне существенного спектра самосопряженного оператора обобщенной модели Фридрихса. Найдено достаточное условие конечности множества точек дискретного спектра. На основании полученной формулы для одной модели Фридрихса доказана бесконечность числа собственных значений на лакуне и получена асимптотика для числа собственных значений.

\section{2. ФОРМУЛИРОВКА ОСНОВНЫХ РЕЗУЛЬТАТОВ}

Пусть $\mathbb{T}^{3}$ - трехмерный тор, т.е. куб $(-\pi, \pi]^{3}$ с отождествлением противоположных граней. Всюду далее операции сложения и умножения на действительное число для элементов множества $\mathbb{T}^{3}=(-\pi, \pi]^{3}$ понимаются как операции в $\mathbb{R}^{3}$ по модулю $(2 \pi \mathbb{Z})^{3}$. Пусть $L_{2}\left(\left(\mathbb{T}^{3}\right)^{2}\right)$ - гильбертово пространство квадратично-интегрируемых функций, определенных на $\left(\mathbb{T}^{3}\right)^{2}$. Рассмотрим трехчастичный решетчатый оператор $H(K), K \in \mathbb{T}^{3}$, который возникает при разложении в прямой интеграл импульсного представления гамильтониана системы трех произвольных квантовых частиц на решетке $\mathbb{Z}^{3}$, взаимодействующих посредством парных короткодействующих потенциалов притяжения [5], [6]. Оператор $H(K), K \in \mathbb{T}^{3}$, действует в гильбертовом пространстве $L_{2}\left(\left(\mathbb{T}^{3}\right)^{2}\right)$ по формуле

$$
H(K)=H_{0}(K)-V, \quad V=V_{1}+V_{2}+V_{3},
$$


где $H_{0}(K)$ является оператором умножения на функцию

$$
\mathcal{E}_{K}(p, q)=\frac{1}{m_{1}} \varepsilon(p)+\frac{1}{m_{2}} \varepsilon(q)+\frac{1}{m_{3}} \varepsilon(K-p-q), \quad \varepsilon(p)=\sum_{i=1}^{3}\left(1-\cos p^{(i)}\right),
$$

$m_{\alpha}>0$ - масса частицы с номером $\alpha$,

$$
\begin{aligned}
& \left(V_{1} f\right)(p, q)=\frac{1}{(2 \pi)^{3 / 2}} \int_{\mathbb{T}^{3}} v_{1}(p-s) f(s, q) d s, \\
& \left(V_{2} f\right)(p, q)=\frac{1}{(2 \pi)^{3 / 2}} \int_{\mathbb{T}^{3}} v_{2}(q-s) f(p, s) d s, \\
& \left(V_{3} f\right)(p, q)=\frac{1}{(2 \pi)^{3 / 2}} \int_{\mathbb{T}^{3}} v_{3}(p-s) f(s, p+q-s) d s,
\end{aligned}
$$

$v_{\alpha}(p)=(2 \pi)^{-3 / 2} \sum_{s \in \mathbb{Z}^{3}} \widehat{v}_{\beta \gamma}(s) e^{i(p, s)}$, а потенциал $\widehat{v}_{\beta \gamma}(s)$ является неотрицательной четной функцией на $\mathbb{Z}^{3}$ и удовлетворяет условию

$$
\lim _{|s| \rightarrow \infty}|s|^{\rho} \widehat{v}_{\beta \gamma}(s)=0, \quad \rho>6, \quad s \in \mathbb{Z}^{3}
$$

(в этих формулах и далее $\{\alpha, \beta, \gamma\}=\{1,2,3\}$ и $|s|=\left|s^{(1)}\right|+\left|s^{(2)}\right|+\left|s^{(3)}\right|$ ).

Обозначим через $A^{1 / 2}$ положительный квадратный корень из оператора $A \geqslant 0$, а через $\sigma(A), \sigma_{\text {disc }}(A)$ и $\sigma_{\text {ess }}(A)$ - спектр, дискретный спектр и существенный спектр оператора $A$ соответственно.

Для существенного спектра $\sigma_{\text {ess }}(H(K))$ оператора $H(K)$ имеет место равенство [5]

$$
\sigma_{\text {ess }}(H(K))=\sigma\left(H_{1}(K)\right) \cup \sigma\left(H_{2}(K)\right) \cup \sigma\left(H_{3}(K)\right), \quad H_{\alpha}(K)=H_{0}(K)-V_{\alpha},
$$

где $K \in \mathbb{T}^{3}, \alpha=1,2,3$. Поскольку

$$
H_{\alpha}(K)-z I=\left(H_{0}(K)-z I\right)\left(I-R_{0}(z) V_{\alpha}\right), \quad R_{0}(z):=\left(H_{0}(K)-z I\right)^{-1},
$$

оператор $\left(I-R_{0}(z) V_{\alpha}\right)^{-1}$ и, следовательно, $W_{\alpha}(z)=\left(I-V_{\alpha}^{1 / 2} R_{0}(z) V_{\alpha}^{1 / 2}\right)^{-1}$ существуют тогда и только тогда, когда $z \notin \sigma\left(H_{\alpha}(K)\right)$, кроме того, $W_{\alpha}(z)$ является положительным оператором при всех $z<\inf \sigma_{\mathrm{ess}}\left(H_{\alpha}(K)\right)$.

При $z<\inf \left\{\sigma\left(H_{1}(K)\right) \cup \sigma\left(H_{2}(K)\right)\right\}, z \notin \sigma\left(H_{3}(K)\right)$ зададим оператор $\mathbf{T}(z)$ в гильбертовом пространстве $L_{2}^{(2)}\left(\left(\mathbb{T}^{3}\right)^{2}\right)$ формулой

$$
\mathbf{T}(z)=\left(\begin{array}{cc}
0 & W_{1}^{1 / 2}(z) K_{12}(z) W_{2}^{1 / 2}(z) \\
W_{2}^{1 / 2}(z) K_{21}(z) W_{1}^{1 / 2}(z) & 0
\end{array}\right)+\left(\begin{array}{ll}
T_{11}(z) & T_{12}(z) \\
T_{21}(z) & T_{22}(z)
\end{array}\right)
$$

где $K_{i j}(z)=V_{i}^{1 / 2} R_{0}(z) V_{j}^{1 / 2}, T_{i j}(z)=W_{i}^{1 / 2}(z) K_{i 3}(z) W_{3}(z) K_{3 j}(z) W_{j}^{1 / 2}(z)$. Заметим, что оператор $W_{3}(z)$ определен при каждом фиксированном $z \notin \sigma\left(H_{3}(K)\right)$, в частности он определен при каждом фиксированном $z$, лежащем на любой лакуне $(a, b)$ спектра $\sigma\left(H_{3}(K)\right)$. Заметим, что за счет оператора $H_{3}(K)$ существенный спектр оператора $H(K)$ также может содержать лакуну [6]. При этом $\mathbf{T}(\cdot)$ как операторная функция определен и на лакуне существенного спектра оператора $H(K)$. 
Пусть $N_{(a, b)}(H(K))$ - число собственных значений оператора $H(K)$ (с учетом кратности), лежащих в интервале $(a, b) \subset \mathbb{R} \backslash \sigma_{\mathrm{ess}}(H(K))$.

Основным результатом настоящей работы является следующая теорема.

Tеорема 1. Число $z_{0}<\inf \left\{\sigma\left(H_{1}(K)\right) \cup \sigma\left(H_{2}(K)\right)\right\}, z_{0} \notin \sigma\left(H_{3}(K)\right)$, является собственным значением оператора $H(K)$ тогда и только тогда, когда функиия $n(1, \mathbf{T}(z))$ имеет разрыв в точке $z=z_{0}$. При этом кратность $k$ собственного значения $z_{0}$ определяется по формуле

$$
k=\lim _{\xi \rightarrow+0}\left[n\left(1, \mathbf{T}\left(z_{0}+\xi\right)\right)-n\left(1, \mathbf{T}\left(z_{0}\right)\right)\right]+\lim _{\xi \rightarrow+0}\left[n\left(1, \mathbf{T}\left(z_{0}-\xi\right)\right)-n\left(1, \mathbf{T}\left(z_{0}\right)\right)\right] .
$$

Мы имеем также два следствия из этой теоремы.

СлеДСТвИЕ 1. Для любого $[a, b] \subset \mathbb{R} \backslash \sigma_{\mathrm{ess}}(H(K)), b<\inf \left\{\sigma\left(H_{1}(K)\right) \cup \sigma\left(H_{2}(K)\right)\right\}$, верно равенство

$$
N_{(a, b)}(H(K))=\bigvee_{a}^{b}(n(1, \mathbf{T}(\cdot))),
$$

где $\bigvee_{a}^{b}(f)$ - полная вариация функиии $f$ на интервале $(a, b)$. При этом из монотонности функции $n(1, \mathbf{T}(\cdot))$ в области $\left(-\infty, \inf \sigma_{\mathrm{ess}}(H(K))\right)$ и из того, что $\lim _{z \rightarrow-\infty}\|\mathbf{T}(z)\|=0$, имеем

$$
N(z)=N_{(-\infty, z)}(H(K))=n(1, \mathbf{T}(z)), \quad z<\inf \sigma_{\mathrm{ess}}(H(K)) .
$$

СлеДСтвие 2. Пусть $(a, b) \subset \mathbb{R} \backslash \sigma_{\mathrm{ess}}(H(K)), b<\inf \left\{\sigma\left(H_{1}(K)\right) \cup \sigma\left(H_{2}(K)\right)\right\}$, $u$ оператор-функиия $\mathbf{T}(z)$ при $z \rightarrow a+0 u z \rightarrow b-0$ сходится равномерно $\kappa$ некоторым операторам $\mathbf{T}(a)$ и $\mathbf{T}(b)$ соответственно. Тогда оператор $H(K)$ на интервале $(a, b)$ может иметь лишь конечное число собственных значений.

\section{3. УРАВНЕНИЕ ФАДДЕЕВА И ПРИНЦИП БИРМАНА-ШВИНГЕРА ДЛЯ ТРЕХЧАСТИЧНОГО ОПЕРАТОРА ШРЕДИНГЕРА}

Основные результаты этого раздела доказываются так же, как в работе [6], где доказательство проведено в случае, когда функции (потенциалы) $v_{1}(\cdot), v_{2}(\cdot)$ и $v_{3}(\cdot)$ являются константами.

ПреДЛОЖеНИЕ 1. Пусть оператор $v_{\alpha}$ действует в $L_{2}\left(\mathbb{T}^{3}\right)$ по формуле

$$
\left(v_{\alpha} f\right)(p)=\int_{\mathbb{T}^{3}} v_{\alpha}(s-p) f(s) d s, \quad f \in L_{2}\left(\mathbb{T}^{3}\right) .
$$

Данный оператор является положительным, и положительный корень $v_{\alpha}^{1 / 2}$ есть интегральный оператор, действующий в $L_{2}\left(\mathbb{T}^{3}\right)$ по формуле

$$
\left(v_{\alpha}^{1 / 2} f\right)(p)=\int_{\mathbb{T}^{3}} \widetilde{v}_{\alpha}(s-p) f(s) d s,
$$

где $\widetilde{v}_{\alpha}(\cdot)$ - некоторая непрерьвная функция на $\mathbb{T}^{3}$. 
ДоказатеЛЬСтво. Рассмотрим оператор $\mathbf{v}_{\alpha}=\mathcal{F}^{-1} v_{\alpha} \mathcal{F}$, унитарно эквивалентный оператору $v_{\alpha}$, где $\mathcal{F}: \ell_{2}\left(\mathbb{Z}^{3}\right) \rightarrow L_{2}\left(\mathbb{T}^{3}\right)$ - стандартное преобразование Фурье:

$$
\begin{array}{rlrl}
(\mathcal{F} \hat{f})(p) & =\frac{1}{(2 \pi)^{3 / 2}} \sum_{s \in \mathbb{Z}^{3}} \hat{f}(s) e^{i(p, s)}, & \hat{f} \in \ell_{2}\left(\mathbb{Z}^{3}\right), \\
\left(\mathcal{F}^{-1} \hat{f}\right)(s) & =\frac{1}{(2 \pi)^{3 / 2}} \int_{\mathbb{T}^{3}} f(p) e^{-i(p, s)} d p, & & f \in L_{2}\left(\mathbb{T}^{3}\right) .
\end{array}
$$

Простое вычисление показывает, что оператор $\mathbf{v}_{\alpha}$ имеет вид

$$
\left(\mathbf{v}_{\alpha} \hat{f}\right)(n)=\hat{v}_{\beta \gamma}(n) \hat{f}(n), \quad\{\alpha, \beta, \gamma\}=\{1,2,3\}, \quad \hat{f} \in \ell_{2}\left(\mathbb{Z}^{3}\right) .
$$

Отсюда и из того, что $\hat{v}_{\beta \gamma}(n) \geqslant 0$ для всех $n \in \mathbb{Z}^{3}$, следует, что $\mathbf{v}_{\alpha}$ является положительным оператором и положительный оператор $\mathbf{v}_{\alpha}^{1 / 2}$ действует по формуле

$$
\left(\mathbf{v}_{\alpha}^{1 / 2} \hat{f}\right)(n)=\sqrt{\hat{v}_{\beta \gamma}(n)} \hat{f}(n), \quad\{\alpha, \beta, \gamma\}=\{1,2,3\}, \quad \hat{f} \in \ell_{2}\left(\mathbb{Z}^{3}\right) .
$$

Заметим, что $v_{\alpha}=\mathcal{F} \mathbf{v}_{\alpha} \mathcal{F}^{-1}$ и $v_{\alpha}^{1 / 2}=\mathcal{F} \mathbf{v}_{\alpha}^{1 / 2} \mathcal{F}^{-1}$. Отсюда, очевидно, вытекает, что оператор $v_{\alpha}^{1 / 2}$ действует в $L_{2}\left(\mathbb{T}^{3}\right)$ по формуле

$$
\left(v_{\alpha}^{1 / 2} f\right)(p)=\int_{\mathbb{T}^{3}} \widetilde{v}_{\alpha}(s-p) f(s) d s, \quad \widetilde{v}_{\alpha}(p)=\frac{1}{(2 \pi)^{3 / 2}} \sum_{s \in \mathbb{Z}^{3}} \sqrt{\hat{v}_{\beta \gamma}(n)} e^{i(p, s)} .
$$

Из условия (1) следует равномерная сходимость ряда в правой части последнего равенства, откуда вытекает непрерывность функции $\widetilde{v}_{\alpha}(\cdot)$ на $\mathbb{T}^{3}$.

ПредЛожение 2. Операторы $V_{1}^{1 / 2}, V_{2}^{1 / 2} u V_{3}^{1 / 2}$ действуют в $L_{2}\left(\left(\mathbb{T}^{3}\right)^{2}\right)$ nо формулам

$$
\begin{aligned}
& \left(V_{1}^{1 / 2} f\right)(p, q)=\frac{1}{(2 \pi)^{3 / 2}} \int_{\mathbb{T}^{3}} \widetilde{v}_{1}(p-s) f(s, q) d s, \\
& \left(V_{2}^{1 / 2} f\right)(p, q)=\frac{1}{(2 \pi)^{3 / 2}} \int_{\mathbb{T}^{3}} \widetilde{v}_{2}(q-s) f(p, s) d s, \\
& \left(V_{3}^{1 / 2} f\right)(p, q)=\frac{1}{(2 \pi)^{3 / 2}} \int_{\mathbb{T}^{3}} \widetilde{v}_{3}(p-s) f(s, p+q-s) d s .
\end{aligned}
$$

ДоКАЗАТЕЛЬСТво. Заметим, что операторы $V_{i}, i=1,2,3$, представляются в виде

$$
V_{1}=v_{1} \otimes I, \quad V_{2}=I \otimes v_{2}, \quad V_{3}=U\left[I \otimes v_{3}\right] U^{-1},
$$

где $I$ - единичный оператор в $L_{2}\left(\mathbb{T}^{3}\right), U$ - унитарный оператор, действующий в $L_{2}\left(\left(\mathbb{T}^{3}\right)^{2}\right)$ по формуле $(U f)(p, q)=f(p, p+q)$. В силу предложения 1 оператор $v_{i}$ является положительным, и оператор $v_{i}^{1 / 2}$ в $L_{2}\left(\mathbb{T}^{3}\right)$ задается формулой $(2)$. Ясно, что

$$
V_{1}^{1 / 2}=v_{1}^{1 / 2} \otimes I, \quad V_{2}=I \otimes v_{2}^{1 / 2}, \quad V_{3}^{1 / 2}=U\left[I \otimes v_{3}^{1 / 2}\right] U^{-1} .
$$

Отсюда легко получить искомое утверждение. 
При $z \notin \sigma_{\mathrm{ess}} H(K)$ зададим следующие операторы $\mathbf{B}(z)$ и $\mathbf{A}(z)$ в гильбертовых пространствах $L_{2}^{(2)}\left(\left(\mathbb{T}^{3}\right)^{2}\right)$ и $L_{2}^{(3)}\left(\left(\mathbb{T}^{3}\right)^{2}\right)$ соответственно:

$$
\begin{aligned}
\mathbf{B}(z)= & \left(\begin{array}{cc}
0 & W_{1}(z) K_{12}(z) \\
W_{2}(z) K_{21}(z) & 0
\end{array}\right)+ \\
& +\left(\begin{array}{lll}
W_{1}(z) K_{13}(z) W_{3}(z) K_{31}(z) & W_{1}(z) K_{13}(z) W_{3}(z) K_{32}(z) \\
W_{2}(z) K_{23}(z) W_{3}(z) K_{31}(z) & W_{2}(z) K_{23}(z) W_{3}(z) K_{32}(z)
\end{array}\right), \\
\mathbf{A}(z)= & \left(\begin{array}{ccc}
0 & W_{1}(z) K_{12}(z) & W_{1}(z) K_{13}(z) \\
W_{2}(z) K_{21}(z) & 0 & W_{2}(z) K_{23}(z) \\
W_{3}(z) K_{31}(z) & W_{3}(z) K_{32}(z) & 0
\end{array}\right) .
\end{aligned}
$$

Поскольку операторы $W_{i}(z), i=1,2,3$, и оператор $R_{0}(z)$ являются аналитическими в $\mathbb{C} \backslash \sigma_{\mathrm{ess}}(H(K))$ (где $\mathbb{C}$ - комплексная плоскость), операторы $\mathbf{A}(z)$ и $\mathbf{B}(z)$ являются аналитическими в $\mathbb{C} \backslash \sigma_{\text {ess }}(H(K))$.

Отметим также, что $\mathbf{A}(z)$ и $\mathbf{B}(z)$ при каждом фиксированном $z \notin \sigma_{\mathrm{ess}} H(K)$ принадлежат классу операторов со следом. Действительно, в силу предложения 2 оператор $V_{j}^{1 / 2} V_{i}^{1 / 2}$ при $i \neq j$ является интегральным оператором, и его ядро по предложению 2 есть непрерывная в $\left(\mathbb{T}^{3}\right)^{2}$ функция. Следовательно, $V_{j}^{1 / 2} V_{i}^{1 / 2}$ принадлежит классу операторов со следом. Так как оператор $R_{0}(z)$ ограничен, $V_{j}^{1 / 2} V_{i}^{1 / 2} R_{0}(z)$ и, следовательно, $K_{i j}(z)=V_{i}^{1 / 2} R_{0}(z) V_{j}^{1 / 2}$ принадлежат классу операторов со следом при $i \neq j$. Далее, из ограниченности операторов $W_{i}(z), i=1,2,3$, следует, что каждый элемент матричных операторов $\mathbf{A}(z)$ и $\mathbf{B}(z)$ принадлежит классу операторов со следом. Таким образом, $\mathbf{A}(z)$ и $\mathbf{B}(z)$ являются операторами со следом при всех $z \notin \sigma_{\mathrm{ess}}(H(K))$.

Лемма 1. Число $z \in \mathbb{C} \backslash \sigma_{\mathrm{ess}}(H(K))$ является собственным значением оператора $H(K)$ тогда и только тогда, когда компактный оператор $\mathbf{B}(z)$ имеет собственное значение, равное единице, причем кратности собственных значений $z$ u 1 совпадают.

ДоказАтельство. Пусть $z \in \mathbb{C} \backslash\left\{\sigma\left(H_{1}(K)\right) \cup \sigma\left(H_{2}(K)\right) \cup \sigma\left(H_{3}(K)\right)\right\}$ - собственное значение оператора $H(K)$ и $f$ - соответствующий собственный вектор, т.е. уравнение $H(K) f=z f$ имеет нетривиальное решение $f$. Так как оператор $R_{0}(z)$ ограничен в $L_{2}\left(\left(\mathbb{T}^{3}\right)^{2}\right)$, имеем $f=R_{0}(z) V f$, где $V=V_{1}+V_{2}+V_{3}$. Отсюда следует, что число $z$ является собственным значением оператора $H(K)$ тогда и только тогда, когда 1 является собственным значением $R_{0}(z) V$, причем кратности $z$ и 1 совпадают.

Отметим, что

$$
R_{0}(z) V=\left(R_{0}(z) V_{1}^{1 / 2}, R_{0}(z) V_{2}^{1 / 2}, R_{0}(z) V_{3}^{1 / 2}\right)\left(\begin{array}{c}
V_{1}^{1 / 2} \\
V_{2}^{1 / 2} \\
V_{3}^{1 / 2}
\end{array}\right) .
$$

Так как для ограниченных операторов $A$ и $B$ каждое ненулевое собственное значение оператора $A B$ является собственным значением $B A$ с той же кратностью (см., 
например, монографию [10]), собственное значение оператора $R_{0}(z) V$, равное единице, является собственным значением оператора $L(z)=V R_{0}(z)$ с той же кратностью. Запишем $L(z)$ в виде

$$
L(z)=\left(\begin{array}{lll}
K_{11}(z) & K_{12}(z) & K_{13}(z) \\
K_{21}(z) & K_{22}(z) & K_{23}(z) \\
K_{31}(z) & K_{32}(z) & K_{33}(z)
\end{array}\right)
$$

и рассмотрим уравнение $L(z) \Phi=\Phi$. Перепишем его как

$$
\operatorname{diag}\left\{I-K_{11}(z), I-K_{22}(z), I-K_{33}(z)\right\} \Phi=\left(\begin{array}{ccc}
0 & K_{12}(z) & K_{13}(z) \\
K_{21}(z) & 0 & K_{23}(z) \\
K_{31}(z) & K_{32}(z) & 0
\end{array}\right) \Phi
$$

Так как $z \notin \bigcup_{i=1}^{3} \sigma\left(H_{i}(K)\right)$, оператор $\operatorname{diag}\left\{I-K_{11}(z), I-K_{22}(z), I-K_{33}(z)\right\}$ обратим, поэтому уравнение (3) можно представить в виде $\Phi=\mathbf{A}(z) \Phi$, т.е. число 1 является собственным значением оператора $\mathbf{A}(z)$, причем совпадают кратности собственных значений $z$ и 1 операторов $H(K)$ и $\mathbf{A}(z)$ соответственно.

Пусть число 1 есть собственное значение оператора $\mathbf{A}(z)$ и $\Phi=\left(\varphi_{1}, \varphi_{2}, \varphi_{3}\right)^{\mathrm{T}}-$ соответствующий собственный вектор, т.е. уравнение

$$
\mathbf{A}(z) \Phi=\Phi
$$

имеет нетривиальное решение $\Phi$. Перепишем это уравнение как систему уравнений

$$
\begin{aligned}
& W_{1}(z) K_{12}(z) \varphi_{2}+W_{1}(z) K_{13}(z) \varphi_{3}=\varphi_{1}, \\
& W_{2}(z) K_{21}(z) \varphi_{1}+W_{2}(z) K_{23}(z) \varphi_{3}=\varphi_{2}, \\
& W_{3}(z) K_{31}(z) \varphi_{1}+W_{3}(z) K_{32}(z) \varphi_{2}=\varphi_{3} .
\end{aligned}
$$

Подставляя выражение для $\varphi_{3}$ из третьего уравнения в первое и второе имеем

$$
\begin{aligned}
& W_{1}(z) K_{12}(z) \varphi_{2}+W_{1}(z) K_{13}(z) W_{3}(z)\left[K_{31}(z) \varphi_{1}+K_{32}(z) \varphi_{2}\right]=\varphi_{1}, \\
& W_{2}(z) K_{21}(z) \varphi_{1}+W_{2}(z) K_{23}(z) W_{3}(z)\left[K_{31}(z) \varphi_{1}+K_{32}(z) \varphi_{2}\right]=\varphi_{2},
\end{aligned}
$$

что эквивалентно уравнению

$$
\mathbf{B}(K, z) \Psi=\Psi, \quad \Psi=\left(\varphi_{1}, \varphi_{2}\right),
$$

т.е. число 1 является собственным значением оператора $\mathbf{B}(z)$.

Наоборот, если 1 - собственное значение, а $\Psi=\left(\varphi_{1}, \varphi_{2}\right)^{\mathrm{T}}$ - соответствующая собственная функция оператора $\mathbf{B}(z)$, то число 1 является собственным значением оператора $\mathbf{A}(z)$, соответствующим собственному вектору $\Phi=\left(\varphi_{1}, \varphi_{2}, \varphi_{3}\right)^{\mathrm{T}}$, где $\varphi_{3}=W_{3}(z) K_{31}(z) \varphi_{1}+W_{3}(z) K_{32}(z) \varphi_{2}$. Легко проверить, что количество линейно независимых векторов, являющихся решениями уравнений (4) и (5), совпадают. Следовательно, кратности собственного значения, равного единице, для операторов $\mathbf{A}(z)$ и $\mathbf{B}(z)$ совпадают. Лемма доказана. 
ЗАмечАниЕ. Уравнение $\mathbf{A}(z) \phi=\phi$ представляет собой аналог уравнения Фаддеева, полученного для трехчастичного непрерывного оператора Шредингера [11].

Оператор-функция $\mathbf{B}(z)$ является аналитическим при

$$
z \in \mathbb{C} \backslash\left\{\sigma\left(H_{1}(K)\right) \cup \sigma\left(H_{2}(K)\right) \cup \sigma\left(H_{3}(K)\right)\right\},
$$

при этом $\mathbf{B}(z)$ для каждого такого $z$ является оператором со следом, поэтому $D(\lambda, z)=\operatorname{det}\left(\mathbf{E}-\lambda^{-1} \mathbf{B}(z)\right)$ определен и аналитичен при $\lambda \neq 0$, где $\mathbf{E}-$ единичный оператор, действующий в $L_{2}^{(2)}\left(\left(\mathbb{T}^{3}\right)^{2}\right)$. Из теоремы XIII.105 в книге [12] вытекает следующая

ЛЕмма 2. Число $\lambda$ является собственным значением оператора $\mathbf{B}(z)$ при $z \in$ $\mathbb{C} \backslash \sigma_{\text {ess }}(H(K))$, тогда и только тогда, когда $D(\lambda, z)=0$.

Из лемм 1 и 2 вытекает следующая

ЛЕмма 3. Число $z \in \mathbb{C} \backslash \sigma_{\mathrm{ess}}(H(K))$ является собственным значением оператора $H(K)$, тогда и только тогда, когда $D(1, z)=0$.

Доказательства следующих двух лемм полностью совпадают с доказательствами, соответственно, леммы 6 и теоремы 2 работы [6]. Лемма 4 отражает известный принцип Бирмана-Швингера.

Лемма 4. Пусть $z<\inf \left\{\sigma\left(H_{1}(K)\right) \cup \sigma\left(H_{2}(K)\right)\right\}, z \notin \sigma\left(H_{3}(K)\right)$. Тогда каждое ненулевое собственное значение оператора $\mathbf{B}(z)$ является собственным значением оператора $\mathbf{T}(z)$ с той же кратностью. При этом число z является собственным значением оператора $H(K)$ тогда и толъко тогда, когда оператор $\mathbf{T}(z)$ имеет собственное значение, равное единице; более того, кратности собственных значений z и 1 совпадают.

Лемма 5. Число $z_{0}<\inf \left\{\sigma\left(H_{1}(K)\right) \cup \sigma\left(H_{2}(K)\right)\right\}, z_{0} \notin \sigma\left(H_{3}(K)\right)$, является регулярной точкой оператора $H$ тогда и только тогда, когда функиия $n(1, \mathbf{T}(\cdot))$ непрерывна в точке $z=z_{0}$.

ПреДЛОЖЕНИЕ 3. Пусть $z_{0} \in \sigma_{\text {disc }}(H(K))$. Тогда для всех малых $\varepsilon>0$ существует $\delta>0$ такое, что имеют место равенства

$$
\begin{aligned}
\operatorname{card}\left\{z \in U_{\delta}: D(1+\varepsilon, z)=0\right\} & =\operatorname{card}\left\{z \in U_{\delta}: D(1-\varepsilon, z)=0\right\}= \\
& =\operatorname{card}\left\{z \in U_{\delta}: D(1, z)=0\right\}
\end{aligned}
$$

где саrd $M-$ мощность множества $M, a U_{\delta}=\left\{z \in \mathbb{C}:\left|z-z_{0}\right|<\delta\right\}-$ дельта-окрестность точки $z_{0}$.

ДоказАТЕльство. В силу леммы $3 D\left(1, z_{0}\right)=0$. Обозначим через $\Gamma_{\delta}$ границу множества $U_{\delta}$. При этом $D(1, z) \neq 0$ для всех $z \in \Gamma_{\delta}$.

Положим

$$
d=\min _{z \in \Gamma_{\delta}}|D(1, z)|, \quad \psi_{\varepsilon}(\lambda)=D(1+\varepsilon, z)-D(1, z), \quad|\varepsilon|<\delta
$$


Так как функция $D(1, z)$ непрерывна, существует число $\rho=\rho(\delta)>0$ такое, что для всех $\varepsilon \in[-\rho, \rho]$ и $z \in \Gamma_{\delta}$ выполняется неравенство $\left|\psi_{\varepsilon}(z)\right|<d$. Таким образом, для каждого фиксированного $\varepsilon \in[-\rho, \rho]$ функции $D(1, z)$ и $\psi_{\varepsilon}(z)$, определенные на $\overline{U_{\delta}}$, удовлетворяют условиям теоремы Руше. Поэтому число лежащих в $U_{\delta}$ нулей функции $D(1, z)$ и число лежащих в $U_{\delta}$ нулей функции $D(1+\varepsilon, z)=\psi_{\varepsilon}(z)+D(1, z)$ совпадают. Предложение доказано.

ПрЕДЛОЖЕНИЕ 4. Предположим, что для малых $\varepsilon>0$ число $\lambda=1 \pm \varepsilon$ является собственным значением оператора $\mathbf{B}(z), z \notin \sigma_{\mathrm{ess}}(H(K))$. Тогда $\operatorname{Im} z=0$.

ДокАЗАтЕЛьство. Пусть $\Psi=\left(\varphi_{1}, \varphi_{2}\right)^{\mathrm{T}} \in L_{2}^{(2)}\left(\mathbb{T}^{3}\right)$ - собственный вектор оператора $\mathbf{B}(z), z \notin \sigma_{\mathrm{ess}}(H(K))$, соответствующий собственному значению $\lambda>0$, т.е.

$$
\begin{aligned}
& \lambda \varphi_{1}=W_{1}(z) K_{12}(z) \varphi_{2}+W_{1}(z) K_{13}(z)\left(W_{3}(z) K_{31}(z) \varphi_{1}+W_{3}(z) K_{32}(z) \varphi_{2}\right), \\
& \lambda \varphi_{2}=W_{2}(z) K_{21}(z) \varphi_{1}+W_{2}(z) K_{23}(z)\left(W_{3}(z) K_{31}(z) \varphi_{1}+W_{3}(z) K_{32}(z) \varphi_{2}\right) .
\end{aligned}
$$

Отсюда, полагая $\varphi_{3}=W_{3}(z) K_{31}(z) \varphi_{1}+W_{3}(z) K_{32}(z) \varphi_{2}$ и учитывая существование оператора $W_{1}(z)^{-1}$ при $z \notin \sigma_{\text {ess }}(H(K))$, имеем

$$
\begin{aligned}
\lambda \varphi_{1} & =\lambda K_{11}(z) \varphi_{1}+K_{12}(z) \varphi_{2}+K_{13}(z) \varphi_{3}, \\
\lambda \varphi_{2} & =K_{21}(z) \varphi_{1}+\lambda K_{22}(z) \varphi_{2}+K_{23}(z) \varphi_{3}, \\
\varphi_{3} & =K_{31}(z) \varphi_{1}+K_{32}(z) \varphi_{2}+K_{33}(z) \varphi_{3} .
\end{aligned}
$$

Последнюю систему запишем в операторной форме

$$
\mathbf{A}_{\lambda} \Phi=\mathbf{K}_{\lambda}(z) \Phi, \quad \Phi=\left(\varphi_{1}, \varphi_{2}, \varphi_{3}\right)^{\mathrm{T}}
$$

Выделяя вещественные и мнимые части функции $\left(\mathcal{E}_{K}(p, q)-z\right)^{-1}$, представим оператор $R_{0}(z)$ в виде $R_{0}(z)=R_{0}^{\prime}(z)+\operatorname{Im} z \cdot R_{0}^{\prime \prime}(z)$, где $R_{0}^{\prime}(z), R_{0}^{\prime \prime}(z)$ - операторы умножения на функции

$$
\frac{\mathcal{E}_{K}(p, q)-\operatorname{Re} z}{\left[\mathcal{E}_{K}(p, q)-\operatorname{Re} z\right]^{2}+(\operatorname{Im} z)^{2}}, \quad \frac{1}{\left[\mathcal{E}_{K}(p, q)-\operatorname{Re} z\right]^{2}+(\operatorname{Im} z)^{2}}
$$

соответственно. Поэтому оператор $\mathbf{K}_{\lambda}(z)$ можно записать через самосопряженные операторы $\mathbf{L}_{\lambda}$ и $\mathbf{M}_{\lambda}$ в виде $\mathbf{K}_{\lambda}(z)=\mathbf{L}_{\lambda}+i \operatorname{Im} z \cdot \mathbf{M}_{\lambda}$, где элементы $M_{i j}$ операторной матрицы $\mathbf{M}_{\lambda}$ действуют в пространстве $L_{2}\left(\left(\mathbb{T}^{3}\right)^{2}\right)$ по формулам

$$
M_{i i}=\lambda V_{i}^{1 / 2} R_{0}^{\prime \prime}(z) V_{i}^{1 / 2}, \quad M_{i j}=V_{i}^{1 / 2} R_{0}^{\prime \prime}(z) V_{j}^{1 / 2} .
$$

Умножая скалярно равенство $\mathbf{A}_{\lambda} \Phi=\mathbf{L}_{\lambda} \Phi+i \operatorname{Im} z \cdot \mathbf{M}_{\lambda} \Phi$ на вектор $\Phi$ и учитывая самосопряженность операторов $\mathbf{A}_{\lambda}, \mathbf{L}_{\lambda}$ и $\mathbf{M}_{\lambda}$, получим

$$
\left(\mathbf{M}_{\lambda} \Phi, \Phi\right) \operatorname{Im} z=0
$$

Поскольку функция $f(\lambda)=\left(\mathbf{M}_{\lambda} \Phi, \Phi\right)$ монотонна и непрерывна, $\left(\mathbf{M}_{1 \pm \varepsilon} \Phi, \Phi\right) \neq 0$ для малых $\varepsilon>0$. Поэтому из равенства (6) имеем $\operatorname{Im} z=0$. 


\section{4. ДОКАЗАТЕЛЬСТВО ОСНОВНОЙ ТЕОРЕМЫ И ЕЕ СЛЕДСТВИЙ}

Доказательство теоремы 1. Пусть $\lambda=1$ есть $k$-кратное собственное значение оператора $\mathbf{T}\left(z_{0}\right)$ и, следовательно, оператора $\mathbf{B}\left(z_{0}\right)$ при $z_{0}<\inf \left\{\sigma_{1}(H(K)) \cup\right.$ $\left.\sigma_{2}(H(K))\right\}, z \notin \sigma_{3}(H(K))$. Функция $\mathbf{B}(z) \Phi$ является векторнозначной аналитической функцией переменной $z$ вблизи точки $z=z_{0}$ для всякого $\Phi \in L_{2}\left(\left(\mathbb{T}^{3}\right)^{2}\right)$. Поэтомy по теореме XII.13 из книги [12] оператор $\mathbf{B}(z)$ имеет в точности $k$ собственных значений $\lambda_{1}(z), \ldots, \lambda_{k}(z)$ (с учетом кратности) в окрестности точки $z=z_{0}$. Все эти собственные значения являются аналитическими функциями в окрестности $z=z_{0}$, причем $\lambda_{i}\left(z_{0}\right)=1$ для всех $i=\overline{1, k}$. Следовательно, оператор $\mathbf{T}(z)$ имеет в точности $k$ собственных значений $\lambda_{1}(z), \ldots, \lambda_{k}(z)$ для вещественного $z$, лежащего вблизи точки $z=z_{0}$.

Лемма 6. Пусть $z_{0} \in \sigma_{\mathrm{disc}}(H(K))$. Тогда для кратности $k$ собственного значения $z_{0}$ справедливо равенство

$$
k=\lim _{\xi \rightarrow 0}\left[n\left(1, \mathbf{T}\left(z_{0}+\xi\right)\right)+n\left(1, \mathbf{T}\left(z_{0}-\xi\right)\right)-2 n\left(1, \mathbf{T}\left(z_{0}\right)\right)\right] .
$$

ДокАзАТЕЛЬСтво. Так как $\mathbf{T}\left(z_{0}\right)$ - компактный оператор, найдется число $\eta_{0}>0$ такое, что $n\left(1, \mathbf{T}\left(z_{0}\right)\right)=n\left(1+\eta_{0}, \mathbf{T}\left(z_{0}\right)\right)$. Отсюда и из неравенства Вейля [13] вытекает, что для малых $|\xi|$ выполняется неравенство

$$
n\left(1+\eta_{0}, \mathbf{T}\left(z_{0}\right)\right) \leqslant n\left(1, \mathbf{T}\left(z_{0}+\xi\right)\right)+n\left(\eta_{0}, \mathbf{T}\left(z_{0}\right)-\mathbf{T}\left(z_{0}+\xi\right)\right)=n\left(1, \mathbf{T}\left(z_{0}+\xi\right)\right) .
$$

Поэтому правая часть равенства (7) неотрицательна.

Пусть $k$ - кратность собственного значения $z_{0}$ оператора $H(K)$. При этом оператор $\mathbf{T}(z)$ имеет в точности $k$ собственных значений $\lambda_{1}(z), \ldots, \lambda_{k}(z)$ (с учетом кратности) при всех $z$ вблизи $z=z_{0}$. В силу предложения 4 , если $\lambda_{1}(z), \ldots, \lambda_{k}(z)$ являются вещественными числами, то $z$ - также вещественное число. Пусть число $\delta$ таково, что для всех $i \in\{1, \ldots, k\}$ имеет место включение $\lambda_{i}\left(z_{0}+\xi\right) \in(1-\delta, 1+\delta)$ при $\xi \in\left(-c_{\delta}, c_{\delta}\right)$. Поскольку кратность нуля определителя $D(1, z)$ в точке $z=z_{0}$ не меньше геометрической кратности собственного значения 1 оператора $\mathbf{T}(z)$, по предложению 3 имеем

$$
\operatorname{card}\left\{z \in\left(-c_{\delta}, c_{\delta}\right): D(1 \pm \varepsilon, z)=0\right\}=\operatorname{card}\left\{z \in\left(-c_{\delta}, c_{\delta}\right): D(1, z)=0\right\}=k+s,
$$

где $s$ - некоторое неотрицательное целое число. Отсюда

$$
\operatorname{card}\left\{i: \lambda_{i}(z)=1-\varepsilon\right\}=\operatorname{card}\left\{i: \lambda_{i}(z)=1+\varepsilon\right\}=k .
$$

Учитывая, что $\lambda_{i}(z) \neq 1$ при $z \neq z_{0}$ и $\lambda_{i}\left(z_{0}\right)=1$, разобьем множество $\{1, \ldots, k\}$ на следующие непересекающиеся множества:

$$
\begin{aligned}
& \{M \nearrow\}=\left\{i: \lambda_{i}\left(z_{0}-c_{\delta}\right)<\lambda_{i}\left(z_{0}\right)<\lambda_{i}\left(z_{0}+c_{\delta}\right)\right\}, \\
& \{M \searrow\}=\left\{i: \lambda_{i}\left(z_{0}-c_{\delta}\right)>\lambda_{i}\left(z_{0}\right)>\lambda_{i}\left(z_{0}+c_{\delta}\right)\right\} .
\end{aligned}
$$


Легко проверить, что

$$
\begin{aligned}
& \operatorname{card}\left\{i: \lambda_{i}\left(z_{0}+\xi\right)>1, \xi \in\left(0, c_{\delta}\right)\right\}=\operatorname{card}\{M \nearrow\} \\
& \operatorname{card}\left\{i: \lambda_{i}\left(z_{0}-\xi\right)>1, \xi \in\left(0, c_{\delta}\right)\right\}=\operatorname{card}\{M \searrow\} .
\end{aligned}
$$

Следовательно,

$$
\operatorname{card}\left\{i: \lambda_{i}\left(z_{0}+\xi\right)>1, \xi \in\left(0, c_{\delta}\right)\right\}+\operatorname{card}\left\{i: \lambda_{i}\left(z_{0}-\xi\right)>1, \xi \in\left(0, c_{\delta}\right)\right\}=k .
$$

С другой стороны

$$
\begin{aligned}
& \operatorname{card}\left\{i: \lambda_{i}\left(z_{0}+\xi\right)>1, \xi \in\left(0, c_{\delta}\right)\right\}=n\left(1, \mathbf{T}\left(z_{0}+\xi\right)\right)-n\left(1+\frac{\delta}{2}, \mathbf{T}\left(z_{0}+\xi\right)\right) \\
& \operatorname{card}\left\{i: \lambda_{i}\left(z_{0}-\xi\right)>1, \xi \in\left(0, c_{\delta}\right)\right\}=n\left(1, \mathbf{T}\left(z_{0}-\xi\right)\right)-n\left(1+\frac{\delta}{2}, \mathbf{T}\left(z_{0}-\xi\right)\right) .
\end{aligned}
$$

Из этих соотношений, учитывая равенства

$$
n\left(1+\frac{\delta}{2}, \mathbf{T}\left(z_{0}-\xi\right)\right)=n\left(1+\frac{\delta}{2}, \mathbf{T}\left(z_{0}+\xi\right)\right)=n\left(1, \mathbf{T}\left(z_{0}\right)\right)
$$

при $|\xi|<c_{\delta}$, получим равенство (7). Лемма доказана.

Доказательство теоремы 1 вытекает из лемм 5 и 6.

Доказательство следствия 1. Пусть оператор $H(K)$ не имеет собственных значений на интервале $(\alpha, \beta)$, где $(\alpha, \beta) \cap \sigma_{\mathrm{ess}}(H(K))=\varnothing$, другими словами, $N_{(\alpha, \beta)}(H(K))=0$. Тогда по лемме 5 имеем, что $n(1, \mathbf{T}(\zeta))=$ const для всех $\zeta \in(\alpha, \beta)$. Отсюда $\bigvee_{\alpha}^{\beta}(n(1, \mathbf{T}(\cdot))=0$. Пусть

$$
\sigma_{\text {disc }}(H(K)) \cap\left(\alpha_{n}, \beta_{n}\right)=\left\{\xi_{n}\right\}, \quad \sigma_{\text {disc }}(H(K)) \cap(\alpha, \beta)=\bigcup_{n}\left\{\xi_{n}\right\} .
$$

Тогда $N_{(\alpha, \beta)}(H(K))=\sum_{n} k_{n}$, где $k_{n}-$ кратность собственного значения $\xi_{n}$. Из теоремы 1 следует, что

$$
\begin{aligned}
\sum_{n} k_{n}= & \sum_{n}\left\{\lim _{\varepsilon \rightarrow+0}\left[n\left(1, \mathbf{T}\left(\xi_{n}+\varepsilon\right)\right)-n\left(1, \mathbf{T}\left(\xi_{n}\right)\right)\right]+\right. \\
& \left.+\lim _{\varepsilon \rightarrow+0}\left[n\left(1, \mathbf{T}\left(\xi_{n}-\varepsilon\right)\right)-n\left(1, \mathbf{T}\left(\xi_{n}\right)\right)\right]\right\}=\bigvee_{\alpha}^{\beta}(n(1, \mathbf{T}(\cdot)) .
\end{aligned}
$$

Доказательство следствия 2. Операторы $\mathbf{T}(a)$ и $\mathbf{T}(b)$ являются компактными, следовательно, при $\zeta=a$ и $\zeta=b$ для некоторого $\delta>0$ имеет место равенство $n(1, \mathbf{T}(\zeta))=n(1+\delta, \mathbf{T}(\zeta))$. В силу непрерывности функции $\|T(\cdot)\|$ существует $\varepsilon>0$ такое, что $\|T(a)-T(z)\|<\delta$ при $z \in[a, a+\varepsilon]$ и $\|T(b)-T(z)\|<\delta$ при $z \in[b-\varepsilon, b]$. Поэтому из неравенства Вейля имеем

$$
\begin{array}{ll}
n(1, \mathbf{T}(a))=n(1+\delta, \mathbf{T}(a)) \leqslant n(1, \mathbf{T}(z)), & z \in[a, a+\varepsilon], \\
n(1, \mathbf{T}(b))=n(1+\delta, \mathbf{T}(b)) \leqslant n(1, \mathbf{T}(z)), & z \in[b-\varepsilon, b],
\end{array}
$$


т.е. функция $n(1, \mathbf{T}(\cdot))$ монотонна на промежутках $(a, a+\alpha)$ и $(b-\beta, b)$. Отсюда

$$
\begin{aligned}
& \bigvee_{a}^{a+\varepsilon}(n(1, \mathbf{T}(\cdot))=n(1, \mathbf{T}(a+\varepsilon))-n(1, \mathbf{T}(a))<\infty, \\
& \bigvee_{b-\varepsilon}^{b}(n(1, \mathbf{T}(\cdot))=n(1, \mathbf{T}(b))-n(1, \mathbf{T}(b-\varepsilon))<\infty .
\end{aligned}
$$

Таким образом, с учетом следствия 1 получаем конечность числа собственных значений $H(K)$, лежащих на промежутке $(a, a+\varepsilon) \cup(b-\varepsilon, b)$. Из аналитичности функции $D(1 ; \cdot)$ в некоторой комплексной окрестности отрезка $[a+\varepsilon, b-\varepsilon]$ получаем конечность множества $\{z \in[a+\varepsilon, b-\varepsilon]: D(1 ; z)=0\}$. Следовательно, по лемме 3 число собственных значений $H(K)$, лежащих на сегменте $[a+\varepsilon, b-\varepsilon]$, конечно.

\section{5. ПРИМЕР}

Рассмотрим трехчастичный решетчатый оператор $H(K)$ в случае, когда массы двух частиц равны, $m_{1}=m_{2}=m$, и $K=\mathbf{0} \in \mathbb{T}^{3}$, а потенциалы притяжения имеют вид

$$
v_{1}(p)=v_{2}(p)=\frac{\mu}{(2 \pi)^{3 / 2}} \sum_{n=0}^{N} \sum_{i=1}^{3} \frac{\cos \left(n p_{j}\right)}{n !}, \quad v_{3}(p)=\mu_{3}(2 \pi)^{3 / 2} \cos p_{1},
$$

где $p \in \mathbb{T}^{3}$, параметры $\mu, \mu_{\alpha}>0$ и $N \leqslant \infty$. При этом преобразование Фурье $\hat{v}_{\beta \gamma}(\cdot)$ функции $v_{\alpha}(\cdot),\{\alpha, \beta, \gamma\}=\{1,2,3\}$, является неотрицательной четной функцией на $\mathbb{Z}^{3}$ и удовлетворяет условию (1). В этом случае трехчастичный решетчатый оператор $H:=H(\mathbf{0})$ действует в $L_{2}\left(\left(\mathbb{T}^{3}\right)^{2}\right)$ по формуле

$$
H=H_{0}-V_{1}-V_{2}-V_{3},
$$

где $H_{0}$ является оператором умножения на функцию

$$
\mathcal{E}(p, q)=\frac{1}{m} \varepsilon(p)+\frac{1}{m} \varepsilon(q)+\frac{1}{m_{3}} \varepsilon(p+q), \quad \varepsilon(p)=\sum_{i=1}^{3}\left(1-\cos p^{(i)}\right)
$$

и

$$
\begin{aligned}
& \left(V_{1} f\right)(p, q)=\frac{\mu}{(2 \pi)^{3}} \sum_{n=0}^{N} \sum_{i=1}^{3} \int_{\mathbb{T}^{3}} \frac{\cos \left(n\left(p_{i}-s_{i}\right)\right)}{n !} f(s, q) d s \\
& \left(V_{2} f\right)(p, q)=\frac{\mu}{(2 \pi)^{3}} \sum_{n=0}^{N} \sum_{i=1}^{3} \int_{\mathbb{T}^{3}} \frac{\cos \left(n\left(q_{i}-s_{i}\right)\right)}{n !} f(p, s) d s \\
& \left(V_{3} f\right)(p, q)=\mu_{3} \int_{\mathbb{T}^{3}} \cos \left(p_{1}-s_{1}\right) f(s, p+q-s) d s .
\end{aligned}
$$

Существенный спектр $\sigma_{\mathrm{ess}}(H)$ оператора $H$ можно представить в виде $\sigma_{\mathrm{ess}}(H)=$ $\sigma\left(H_{1}\right) \cup \sigma\left(H_{3}\right)[5]$. Здесь спектр оператора $H_{\alpha}=H_{0}-V_{\alpha}$ есть

$$
\sigma\left(H_{\alpha}\right)=\left[m_{0}, M_{0}\right] \cup \bigcup_{k \in \mathbb{T}^{3}}\left\{z+\frac{1}{m_{\alpha}} \varepsilon(k): z \in \sigma_{\text {disc }}\left(h_{\alpha}(k)\right)\right\},
$$


где $m_{0}=\min _{p, q} \mathcal{E}(p, q), M_{0}=\max _{p, q} \mathcal{E}(p, q)$, а оператор $h_{\alpha}(k)$ действует в гильбертовом пространстве $L_{2}\left(\mathbb{T}^{3}\right)$ по формуле $h_{\alpha}(k)=h_{0}^{(\alpha)}(k)-\mathbf{v}_{\alpha}$, где $h_{0}^{(\alpha)}(k)$ - оператор умножения на функцию

$$
\mathcal{E}_{k}^{(\alpha)}(p)=\frac{1}{m_{\beta}} \varepsilon(p)+\frac{1}{m_{\gamma}} \varepsilon(p-k), \quad k \in \mathbb{T}^{3},
$$

и $\mathbf{v}_{\alpha}$ - интегральный оператор с ядром $v_{\alpha}(p-s)$.

Заметим, что $V_{\alpha}^{1 / 2} R_{0}(z) V_{\alpha}^{1 / 2}=\mu \widetilde{V}_{\alpha}^{1 / 2} R_{0}(z) \widetilde{V}_{\alpha}^{1 / 2}$, где $\widetilde{V}_{\alpha}=\mu^{-1} V_{\alpha}, R_{0}(z)$ - оператор умножения на функцию $(\mathcal{E}(p, q)-z)^{-1}, \alpha=1,2$. Из невырожденности точки минимума функции $\mathcal{E}(\cdot, \cdot)$ следует, что операторнозначная функция $\widetilde{V}_{\alpha}^{1 / 2} R_{0}(\cdot) \widetilde{V}_{\alpha}^{1 / 2}$ является непрерывной на полупрямой $(-\infty, 0]$ в операторной топологии. Положим

$$
\mu^{*}=\max _{z \leqslant 0}\left\|\widetilde{V}_{\alpha}^{1 / 2} R_{0}(z) \widetilde{V}_{\alpha}^{1 / 2}\right\|=\left\|\widetilde{V}_{\alpha}^{1 / 2} R_{0}(0) \widetilde{V}_{\alpha}^{1 / 2}\right\|
$$

Для $\alpha=2$ при $\mu \in\left[0, \mu^{*}\right)$ и $z \leqslant 0$ справедливо неравенство $\left\|V_{\alpha}^{1 / 2} R_{0}(z) V_{\alpha}^{1 / 2}\right\|<1$. Поэтому оператор $W_{\alpha}(z)=\left(I-V_{\alpha}^{1 / 2} R_{0}(z) V_{\alpha}^{1 / 2}\right)^{-1}$ существует и является положительным оператором при всех $z \leqslant 0$. Отсюда получим $\sigma\left(H_{1}\right)=\sigma\left(H_{2}\right)=\left[0, M_{0}\right]$.

$\mathrm{C}$ целью описания спектра $H_{3}$ изучим спектральные свойства оператора $h_{3}(k)$. Пусть $\lambda>6 / m_{3}$. Положим $\mu_{3}^{*}=\left(4 \pi^{3}\right)^{-1}\left(6 / m_{3}+\lambda\right)$. Оператор $h_{3}(k)$ действует в $L_{2}\left(\mathbb{T}^{3}\right)$ по формуле

$$
\left(h_{3}(k) f\right)(p)=\left(\frac{1}{m} \varepsilon(p)+\frac{1}{m} \varepsilon(p-k)\right) f(p)-\mu_{3} \int_{\mathbb{T}^{3}} \cos \left(p_{1}-s_{1}\right) f(s) d s .
$$

Лемма 7. Пусть $\mu_{3}>\mu_{3}^{*}$. Тогда для всех $k \in \mathbb{T}^{3}$ дискретный спектр операmора $h_{3}(k)$ состоит из двух точек, $\sigma_{\text {disc }}\left(h_{\alpha}(k)\right)=\left\{\lambda_{1}(k), \lambda_{2}(k)\right\}$, где $\lambda_{1}(\cdot)$ u $\lambda_{2}(\cdot)$ являются непрерывными функииями, $-\lambda>\lambda_{1}(k) \geqslant \lambda_{2}(k)$, причем

$$
\begin{aligned}
\lambda_{1}(\mathbf{0}) & =\min _{k \in \mathbb{T}^{3}} \lambda_{1}(k)<\lambda_{2}(\mathbf{0})=\min _{k \in \mathbb{T}^{3}} \lambda_{2}(k), \\
\lambda_{1}\left(k^{0}\right) & =\max _{k \in \mathbb{T}^{3}} \lambda_{1}(k)=\max _{k \in \mathbb{T}^{3}} \lambda_{2}(k)=\lambda_{2}\left(k^{0}\right),
\end{aligned}
$$

где $k^{0} \in \Pi=\left\{k=\left(k_{1}, k_{2}, k_{3}\right):\left|k_{1}\right|=\left|k_{2}\right|=\left|k_{3}\right|=\pi\right\}$. Cnектр оператора $H_{3}$ есть $\sigma\left(H_{3}\right)=\left[0, M_{0}\right] \cup\left[\tau^{\prime}, \tau^{\prime \prime}\right]$, где $\tau^{\prime}=\lambda_{1}(\mathbf{0}), \tau^{\prime \prime}=\lambda_{1}\left(k^{0}\right)+6 / m_{3}$.

ДокАЗАтЕЛьство. Пусть $z \in \mathbb{C} \backslash \sigma_{\mathrm{ess}}\left(h_{3}(k)\right)$ - собственное значение оператора $h_{3}(k)$ и $f$ - соответствующий собственный вектор, т.е. уравнение $h_{3}(k) f=z f$ имеет нетривиальное решение $f$. Отсюда

$$
f(p)=\frac{\mu_{3}}{\mathcal{E}_{k}^{(\alpha)}(p)-z}\left(c_{1} \cos p_{1}+c_{2} \sin p_{1}\right),
$$

где

$$
c_{1}=\int_{\mathbb{T}^{3}} \cos s_{1} \cdot f(s) d s, \quad c_{2}=\int_{\mathbb{T}^{3}} \sin s_{1} \cdot f(s) d s .
$$


Подставляя выражение (9) в (10), получим линейную систему для $c_{1}, c_{2}$ :

$$
\begin{aligned}
& \left(1-\mu_{3} \int_{\mathbb{T}^{3}} \frac{\cos ^{2} s_{1} d s}{\mathcal{E}_{k}^{(\alpha)}(s)-z}\right) c_{1}-\mu_{3} \int_{\mathbb{T}^{3}} \frac{\cos s_{1} \sin s_{1} d s}{\mathcal{E}_{k}^{(\alpha)}(s)-z} c_{2}=0, \\
& -\mu_{3} \int_{\mathbb{T}^{3}} \frac{\cos s_{1} \sin s_{1} d s}{\mathcal{E}_{k}^{(\alpha)}(s)-z} c_{1}+\left(-\mu_{3} \int_{\mathbb{T}^{3}} \frac{\cos ^{2} s_{1} d s}{\mathcal{E}_{k}^{(\alpha)}(s)-z}\right) c_{2}=0 .
\end{aligned}
$$

Отсюда легко получить, что число $z \in \mathbb{C} \backslash \sigma_{\mathrm{ess}}\left(h_{3}(k)\right)$ является собственным значением оператора $h_{3}(k)$ тогда и только тогда, когда $\Delta(k ; z)=0$, где $\Delta(k ; z)$ - определитель матрицы системы (11). Совершая в каждом интеграле системы (11) замену переменной $s=t+k / 2$, получим $\Delta(k ; z)=\Delta_{1}(k ; z) \Delta_{2}(k ; z)$, где

$$
\begin{aligned}
& \Delta_{1}(k ; z)=1-\mu_{3} \int_{\mathbb{T}^{3}} \frac{\cos ^{2} t_{1} d t}{m^{-1} \varepsilon(t+k / 2)+m^{-1} \varepsilon(t-k / 2)-z}, \\
& \Delta_{2}(k ; z)=1-\mu_{3} \int_{\mathbb{T}^{3}} \frac{\sin ^{2} t_{1} d t}{m^{-1} \varepsilon(t+k / 2)+m^{-1} \varepsilon(t-k / 2)-z} .
\end{aligned}
$$

Простое вычисление показывает, что при каждом $z<0$ множеством стационарных точек функции $\Delta_{\alpha}(\cdot ; z), \alpha=1,2$, является множество $\{\boldsymbol{0}\} \cup \Pi$. Вычисляя вторые частные производные функции $\Delta_{\alpha}(\cdot ; z)$, получим

$$
\frac{\partial^{2}}{\partial k_{i}^{2}} \Delta_{\alpha}(\mathbf{0} ; z)>0, \quad \frac{\partial^{2}}{\partial k_{i} \partial k_{j}} \Delta_{\alpha}(\mathbf{0} ; z)=0, \quad i \neq j
$$

а при $k^{0} \in \Pi$

$$
\frac{\partial^{2}}{\partial k_{i}^{2}} \Delta_{\alpha}\left(k^{0} ; z\right)<0, \quad \frac{\partial^{2}}{\partial k_{i} \partial k_{j}} \Delta_{\alpha}\left(k^{0} ; z\right)=0, \quad i \neq j .
$$

Поэтому при каждом $z<0$ имеют место равенства

$$
\Delta_{\alpha}(\mathbf{0} ; z)=\min _{k \in \mathbb{T}^{3}} \Delta_{\alpha}(k ; z), \quad \Delta_{\alpha}\left(k^{0} ; z\right)=\max _{k \in \mathbb{T}^{3}} \Delta_{\alpha}(k ; z), \quad k^{0} \in \Pi, \quad \alpha=1,2 .
$$

Пользуясь монотонностью функции $\Delta_{\alpha}(k ; z)$ по $z$ для каждого $k \in \mathbb{T}^{3}$ и всех $z<-\lambda$, получим для таких $z$ и $k^{0} \in \Pi$

$$
\Delta_{\alpha}(k ; z) \leqslant \Delta_{\alpha}\left(k^{0} ; z\right)=1-\mu_{3} \frac{4 \pi^{3}}{6 / m_{3}-z}<1-\mu_{3}^{*} \frac{4 \pi^{3}}{6 / m_{3}+\lambda}=0 .
$$

В силу того что $\lim _{z \rightarrow-\infty} \Delta_{\alpha}(k ; z)=1$, существует число $z_{\alpha}<-\lambda$ такое, что $\Delta_{\alpha}\left(k ; z_{\alpha}\right)>0$. Так как $\Delta_{\alpha}(k ; \cdot)$ - непрерывная функция (по $\left.z\right)$, принимающая на концах сегмента $\left[z_{\alpha},-\lambda\right]$ значения разных знаков, она обращается в нуль в некоторой точке $\lambda_{\alpha}(k) \in\left(z_{\alpha},-\lambda\right)$, которая в силу монотонности функции единственна. Следовательно, оператор $h_{3}(k)$ имеет только два собственных значения, меньших $-\lambda$. Функция $\Delta_{\alpha}(k ; z)$ является аналитической в $\mathbb{T}^{3} \times(-\infty, 0)$, поэтому функция $\lambda_{\alpha}(\cdot)$ непрерывна в $\mathbb{T}^{3}, \alpha=1,2$. 
Поскольку $\Delta_{\alpha}(\mathbf{0} ; \lambda(k))<\Delta_{\alpha}(k ; \lambda(k))=0$ при $k \neq \mathbf{0}$, мы имеем такую оценку: $\Delta_{\alpha}(\mathbf{0} ; \lambda(k))<\Delta_{\alpha}(\mathbf{0} ; \lambda(\mathbf{0}))=0$. Пользуясь монотонностью функции $\Delta_{\alpha}(k ; z)$ по $z$, получим $\lambda_{\alpha}(\mathbf{0})=\min _{k \in \mathbb{T}^{3}} \lambda_{\alpha}(k)$. Аналогично $\lambda_{\alpha}\left(k^{0}\right)=\max _{k \in \mathbb{T}^{3}} \lambda_{\alpha}(k)$ при $k^{0} \in \Pi$. Из неравенства

$$
\int_{-\pi}^{\pi} \frac{\sin ^{2} \rho d \rho}{m^{-1}(2-\cos (x / 2) \cos \rho)+a^{2}}<\int_{-\pi}^{\pi} \frac{\cos ^{2} \rho d \rho}{m^{-1}(2-\cos (x / 2) \cos \rho)+a^{2}},
$$

верного при $x \in[-\pi, \pi], x \neq 0, a \neq 0$, следует $\Delta_{1}(k ; z)<\Delta_{2}(k ; z)$. Отсюда вытекает, что $\lambda_{1}(\mathbf{0})<\lambda_{2}(\mathbf{0})$. Так как $\Delta_{1}\left(k^{0} ; z\right)=\Delta_{2}\left(k^{0} ; z\right)$ при $k^{0} \in \Pi$, имеем $\lambda_{1}\left(k^{0}\right)=\lambda_{2}\left(k^{0}\right)$.

Из леммы 7 согласно соотношению (8) получим $\sigma\left(H_{3}\right)=\left[\tau^{\prime}, \tau^{\prime \prime}\right] \cup\left[0, M_{0}\right]$. Лемма доказана.

Простое вычисление показывает, что $V_{3}^{1 / 2}=2^{-1} \mu_{3}^{-1 / 2} \pi^{-3 / 2} V_{3}$. Пользуясь методами теории линейных интегральных уравнений, докажем следующую лемму.

Лемма 8. Для каждого $z \notin \sigma\left(H_{3}\right)$ оператор $W_{3}(z)=\left(I-V_{3}^{1 / 2} R_{0}(z) V_{3}^{1 / 2}\right)^{-1}$ существует и действует в $L_{2}\left(\left(\mathbb{T}^{3}\right)^{2}\right)$ по формуле

$$
\begin{aligned}
\left(W_{3}(z) f\right)(p, q) & =f(p, q)+ \\
+ & \frac{\mu_{3}}{4 \pi^{3}} \frac{a_{1}(p+q ; z) \cos \left(\left(p_{1}-q_{1}\right) / 2\right)}{\Delta_{1}\left(p+q ; z-m_{3}^{-1} \varepsilon(p+q)\right)} \int_{\mathbb{T}^{3}} \cos \left(s_{1}-\frac{p_{1}+q_{1}}{2}\right) f(s) d s+ \\
+ & \frac{\mu_{3}}{4 \pi^{3 / 2}} \frac{a_{2}(p+q ; z) \sin \left(\left(p_{1}-q_{1}\right) / 2\right)}{\Delta_{2}\left(p+q ; z-m_{3}^{-1} \varepsilon(p+q)\right)} \int_{\mathbb{T}^{3}} \sin \left(s_{1}-\frac{p_{1}+q_{1}}{2}\right) f(s) d s
\end{aligned}
$$

¿əe

$$
\begin{aligned}
& a_{1}(p+q ; z)=\int_{\mathbb{T}^{3}} \frac{\cos ^{2} s d s}{m^{-1} \varepsilon(s)+m^{-1} \varepsilon(s-p-q)+m^{-1} \varepsilon(p+q)-z}, \\
& a_{2}(p+q ; z)=\int_{\mathbb{T}^{3}} \frac{\sin ^{2} s d s}{m^{-1} \varepsilon(s)+m^{-1} \varepsilon(s-p-q)+m^{-1} \varepsilon(p+q)-z} .
\end{aligned}
$$

Так как точка $\mathbf{0}$ (точка $k^{0} \in \Pi$ ) является точкой невырожденного минимума (максимума) для функции $\Delta_{\alpha}\left(\cdot ; \lambda_{\alpha}(\mathbf{0})\right)$ (функции $\Delta_{\alpha}\left(\cdot ; \lambda_{\alpha}\left(k^{0}\right)\right)$ ), точка $p+q=\mathbf{0}$ (точка $p+q=k^{0} \in \Pi$ ) является точкой невырожденного минимума (максимума) функции $\Delta_{\alpha}\left(p+q ; \tau^{\prime}-m_{3}^{-1} \varepsilon(p+q)\right)$ (функции $\left.\Delta_{\alpha}\left(p+q ; \tau^{\prime \prime}-m_{3}^{-1} \varepsilon(p+q)\right)\right)$. Отсюда с учетом леммы 8 вытекает, что операторы $W_{3}\left(\tau^{\prime}\right)$ и $W_{3}\left(\tau^{\prime \prime}\right)$ ограничены.

Отметим, что оператор $\mathbf{T}(z)$, соответствующий оператору $H$, определен на $\left(-\infty, \tau^{\prime}\right) \cup\left(\tau^{\prime \prime}, 0\right)$. Покажем, что оператор $\mathbf{T}(z)$ является компактным при $z=\tau^{\prime}$, $z=\tau^{\prime \prime}$ и $z=0$. Из невырожденности точки $(\mathbf{0}, \mathbf{0})$ минимума функции $\mathcal{E}(\cdot, \cdot)$ следует, что оператор $K_{i j}(0)=V_{i}^{1 / 2} R_{0}(0) V_{j}^{1 / 2}$ при $i \neq j$ является компактным. Отсюда, заметив компактность операторов $K_{i j}(z), i \neq j$, при $z=\tau^{\prime}$ и $z=\tau^{\prime \prime}$, а также ограниченность операторов $W_{i}(z), i=1,2,3$, при $z=\tau^{\prime}, z=\tau^{\prime \prime}$ и $z=0$, получим, что каждый элемент матричного оператора $\mathbf{T}(z)$ является компактным при $z=\tau^{\prime}$, $z=\tau^{\prime \prime}$ и $z=0$. Таким образом, в силу следствия 1 из теоремы 1 справедлива следующая 
ТЕОрема 2. Для всех $\mu \leqslant \mu^{*} u \mu_{3}>\mu_{3}^{*}$ существенный спектр $\sigma_{\mathrm{ess}}(H)$ оператора $H$ содержит лакуны: $\sigma_{\mathrm{ess}}(H)=\left[\tau^{\prime}, \tau^{\prime \prime}\right] \cup\left[0, M_{0}\right], \tau^{\prime}<\tau^{\prime \prime}<0$, а дискретный спектр $\sigma_{\mathrm{disc}}(H)$ оператора $H$ является конечным множеством.

Предположим, что $N=0$ и $\mu=\mu^{0} / 3$. В этом случае потенциалы $v_{1}$ и $v_{2}$ являются контактными и $\sigma\left(H_{1}\right)=\sigma\left(H_{2}\right)=\left[0, M_{0}\right]$, а операторы $h_{1}(\mathbf{0})$ и $h_{2}(\mathbf{0})$ имеют виртуальные уровни в нуле. Пользуясь теоремой 1 и учитывая компактность операторов $T_{i j}(0), i, j=1,2$, совершенно аналогично тому, как это сделано в работе [6], мы можем доказать следующую теорему.

Теорема 3. Пусть $\mu_{3}>\mu_{3}^{*} u \mu=\mu^{0} / 3$. Тогда оператор $H$ на лакуне $\left(\tau^{\prime \prime}, 0\right)$ существенного спектра имеет бесконечное число собственных значений.

Благодарности. Автор выражает благодарность рецензенту за полезные замечания. Данная работа была поддержана TUBITAK (Турция).

\section{Список литературы}

[1] A. V. Sobolev, Comm. Math. Phys., 156:1 (1993), 101-126.

[2] H. Tamura, Nagoya Math. J., 130 (1993), 55-83.

[3] С. Н. Лакаев, З.Э. Муминов, Функи. анализ и его прил., 37:3 (2003), 80-84.

[4] Ж. И. Абдуллаев, С. Н. Лакаев, ТМФ, 136:2 (2003), 231-245.

[5] S. Albeverio, S. N. Lakaev, Z. I. Muminov, Math. Nachr., 280:7 (2007), 699-716; arXiv: math-ph/0312050.

[6] М.Э. Муминов, ТМФ, 159:2 (2009), 299-317.

[7] С. Н. Лакаев, М.Э. Муминов, ТМФ, 135:3 (2003), 478-503.

[8] А. А. Владимиров, Матем. заметки, 74:6 (2003), 838-847.

[9] М.Э. Муминов, Матем. заметки, 82:1 (2007), 75-83.

[10] П. Халмош, Гильбертово пространство в задачах, Мир, М., 1970.

[11] Л. Д. Фаддеев, Математические вопросы квантовой теории рассеяния для системь mpех частии, Тр. МИАН СССР, 69, ред. И. Г. Петровский, Изд-во АН СССР, М.-Л., 1963.

[12] М. Рид, Б. Саймон, Методы современной математической физики, т. 4, Анализ операторов, Мир, М., 1982.

[13] М.Ш. Бирман, М.З. Соломяк, Спектральная теория самосопряженных операторов в гилъбертовом пространстве, Изд-во ЛГУ, Л., 1980.

Поступила в редакцию 13.11.2009, после доработки 16.12.2009 